\title{
EL SENTIDO DE VIDA Y LOS VALORES INTERPERSONALES EN UNIVERSITARIOS ESTUDIANTES DE LA CARRERA DE PSICOLOGÍA
}

\author{
The sense of life and the interpersonal values in university \\ students of the career of Psychology
}

Manuel Arboccó de los Heros*

\begin{abstract}
Resumen
El presente artículo es un resumen de la investigación que realizamos con el propósito de identificar si existe relación entre el nivel de sentido de vida y los valores interpersonales en universitarios estudiantes de la carrera de Psicología de una universidad privada de Lima. Además comparamos si estas dos variables se diferencian en función al tiempo de estudios del alumno y a su sexo.

Se realizó una investigación de tipo correlacional y bajo un diseño transversal comparativo. La muestra estuvo conformada por 277 estudiantes del sistema presencial matriculados en el semestre 2017 en la Facultad de Psicología de una universidad privada de Lima, de asistencia regular y con consentimiento de participar en la investigación. Estos estudiantes corresponden a tres periodos de la carrera: Primer año de estudios (ciclos Iy II); Tercer año de estudios (ciclos Vy VI) y el 5to. Año de estudios (Internado I y II - realizando prácticas pre-profesionales).La investigación se llevó a cabo, utilizando el Cuestionario de Valores Interpersonales SIV de Leonard Gordon y el Inventario de propósito en la vida desarrollado por James Crumbaugh y Leonard Matholick, ambas pruebas presentan confiabilidad y validez. Se concluyó que existe una relación significativa entre el sentido de vida y los valores interpersonales en el grupo de estudiantes de la carrera de Psicología.
\end{abstract}

Palabras clave: Sentido de vida, valores interpersonales, soporte, conformidad, reconocimiento, independencia, benevolencia, liderazgo, crecimiento personal, salud psicológica, estudiantes de Psicología.

\begin{abstract}
This article is a summary of the research we conducted in order to identify if there is a relationship between the level of sense of life and interpersonal values in university students of psychology from a private university in Lima. We also compare if these two variables will differ in function to the time of the student's studies and their gender.

A correlational type research was carried out under a comparative/transversal design. The sample was composed by 277 face-to-face system students enrolled in the 2017 semester in the Psychology School from a private university in Lima, of regular attendance and with consent to participate in the research. These students correspond to three periods of the career: first year of studies (cycles I and II); third year of studies (cycles V and VI) and fifth year of studies (internship I and II - doing their pre professional practices)The research was carried out using Leonard Gordon's Survey of Interpersonal Values (SIV) and the James Crumbaugh and Leonard Matholick's Purpose in Life tes, both tests present reliability and validity. It was concluded that there is a significant relationship between the sense of life and interpersonal values in the group of students of the Psychology career.
\end{abstract}

Keywords: Sense of life, interpersonal values, support, compliance, recognition, independence, benevolence, leadership, personal growth, psychological health, Psychology students.

\footnotetext{
* Psicólogo y psicoterapeuta. Docente en la Universidad Femenina del Sagrado Corazón y en la Universidad Inca Garcilaso de la Vega.manoloarbocco@gmail.com
} 


\section{INTRODUCCIÓN}

Sentido de vida y valores interpersonales, dos importantes aspectos humanos que consideramos hoy para elaborar esta investigación que presentamos a continuación, y eligiendo como muestra de estudio a un grupo de estudiantes de la carrera de Psicología de una universidad privada de la ciudad de Lima, porque consideramos que es oportuno indagar sobre la realidad psíquica, social y espiritual de los que toman a la Psicología por vocación y carrera.

Tiempos difíciles los que nos tocan ahora; el estrés, el acoso escolar, la corrupción, la violencia en todas sus formas y los vacíos vitales se presentan continuamente. De ahí el interés en elegir variables como el propósito de vida y los valores en la esfera de lo interpersonal para estudiarlas detenidamente. Buscaremos conocer, por eso, el nivel de sentido de vida y los valores interpersonales en un grupo de estudiantes de Psicología e identificar si existe relación entre ambos aspectos así como conocer si hay diferencias por aspectos como el sexo y el año de estudios cursados.

\section{PLANTEAMIENTO DEL PROBLEMA}

Los psicólogos, como otros profesionales, debemos contar con ciertas características personales que nos permitan un ejercicio de la carrera dentro de lo más óptimo posible. Algunas de estas características (o rasgos como mencionan algunos psicólogos) de pronto están presentes al ingresar a la facultad o bien, van trabajándose y desarrollándose con el paso del tiempo de estudios. Características que poco a poco van definiendo un perfil del estudiante y del profesional de la Psicología.

Siempre que hablamos de salud psicológica, nos preguntamos si nosotros los profesionales y los estudiantes de la Psicología la hemos alcanzado, cuando a veces nos enteramos de problemas donde están inmersos estudiantes y hasta colegas. Se hace necesario evaluar al estudiante y al interno de Psicología y revisar aspectos personales como sus valores, su sentido de vida, su compromiso con la carrera, sus relaciones interpersonales, su inteligencia social y emocional; entre otras variables psicológicas. Ser un(a) psicólogo(a) implica necesariamente un nivel de madurez, interés por el prójimo, adecuadas relaciones interpersonales, sentido del humor, buen nivel intelectual, estabilidad emocional, una buena autoestima, capacidad de trabajo, entre otros aspectos. Preocupados por no siempre conseguir estos aspectos, decidimos hacer una investigación para evaluar y así conocer tanto el sentido de vida como los valores interpersonales en un grupo de estudiantes de Psicología, dividiéndolos en tres segmentos: estudiantes del primer año de estudios (I y II ciclo de estudios), de tercer año (estudiantes de V y VI ciclo de estudios) e internos de Psicología (estudiantes que realizan las prácticas pre profesionales en hospitales, colegios y empresas).

Valores interpersonales como el liderazgo, la benevolencia, la independencia, el apoyo al prójimo son aspectos que bien deben estar presentes en estudiantes de Psicología y más aún en internos de esa carrera. Cierto es, que la práctica de una carrera puede generar algunos cambios en estos aspectos, como vemos en investigaciones que indican que el valor de la benevolencia va disminuyendo en médicos desde que ingresan jóvenes a su práctica hasta que alcanzan ya algún tiempo de ejercicio profesional, sobre todo en ciertos ambientes como pueden ser hospitales públicos (Leonard Gordon e Iván Mensh, 1962, citados por Smith, 1985 y por Lobos, 1992). Esto es interesante, porque es vox populi, el reclamo que se les hace a algunos galenos por considerarlos fríos y distantes en su trato. Esperemos no pase eso también entre los psicólogos, pero eso ya sería tema de otra investigación.

El sistema de valores es un parámetro importante en las Ciencias Sociales si se toma en cuenta que "el sistema de valores subyace a las actitudes y puede convertirse en un activador de la conducta" (Chocano, 1990; citado por Lobos, 1992). Por su parte, el sentido o propósito de vida es la manera como cada persona organiza su existencia con una mirada de su presente así como hacia el futuro, es decir con una prospectiva (Arboccó, 2015). El sentido de vida, que no es solo algo racional sino afectivo también (Martínez, 2013) precisa de objetivos, valoraciones, metas, sueños y percepciones de uno mismo, el mundo y el futuro. Abarca una respuesta al por qué de nuestra existencia y sobre todo al para qué de la 
misma. Consideramos que un buen nivel de sentido de nuestra vida y sólidos valores interpersonales son dos aspectos importantísimos en el bienestar de cualquier persona, y más aún en el estudiante de la Psicología. Al ser una carrera donde trabajamos con personas y el desgaste es fuerte, un interno y más aún un profesional de la Psicología requiere estar bien parado frente a ciertos problemas que por su papel de psicólogo no está necesariamente indemne; hablamos del estrés, la fatiga, el cansancio, el ahora llamado "Síndrome de Burnout", entre otras dificultades a las que podemos estar expuestos en una sociedad complicada como la que nos toca vivir estos días.

Entonces, conforme a lo planteado, esta investigación pretende dar respuesta a las siguientes preguntas.

\section{Problema principal:}

1. ¿Existirá relación entre las variables Sentido de vida y Valores interpersonales en estudiantes de la carrera de Psicología de una universidad privada de Lima?

\section{Problemas específicos:}

2. ¿Cuáles son los niveles de Sentido de vida que presentan los estudiantes de la carrera de Psicología?

3. ¿Son estos niveles diferentes según el sexo de los estudiantes?

4. ¿Existen diferencias en estos niveles a lo largo de los años de estudio de la carrera?

5. ¿Cuáles son los valores interpersonales que caracterizan a los estudiantes de la carrera de Psicología?

6. ¿Qué relación existe entre el Sentido de vida y los Valores interpersonales en los estudiantes de la carrera de Psicología?

\section{JUSTIFICACIÓN}

Los docentes universitarios, vemos estudiantes que no siempre llegan con bienestar psicológico a la Facultad. Difícil en algunos casos, más que en otros, por una diversidad de circunstancias que les toca atravesar a los adolescentes, además de vivir hoy en una sociedad que muchas veces promueve la desconfianza, el individualismo y la adicción tecnológica (Arboccó, 2015). ¿Cómo, pues, podemos desarrollar salud psicológica en una sociedad que para muchos anda degradándose? Las neurosis histéricas del siglo XIX han dado paso a una variedad de síntomas vagos y un vacío existencial que cada vez crece más y es campo de cultivo de una serie de patologías. ¿Cómo fortalecer nuestros valores interpersonales? Cuando vivimos épocas de miedo, de violencia, de competitividad salvaje muchas veces y con un nivel de corrupción política inmenso que deja como mensaje la poca preocupación por el otro y una fragilidad en temas morales y por supuesto sociales.

Por eso consideramos interesante y necesario hacer una evaluación de cómo llegan, cómo transcurren y cómo terminan la carrera de Psicología, un grupo de estudiantes de ambos sexos, en temas interpersonales y de sentido de vida. Una medida de cómo llegan puede servir para conocer más el perfil de este ingresante del siglo XXI y compararlo con el perfil que se desea en las diferentes casas de estudios donde se imparte Psicología, también. Hasta qué punto vamos a requerir una malla curricular que disponga de espacios para trabajar con los estudiantes aspectos sociales y personales, con el fin de que el estudiante de Psicología vaya percatándose de su propia conducta y luego ponga manos a la obra en el mejoramiento de su propia persona. Recién ahí podrá fortalecerse y salir al campo laboral para intentar colaborar a la mejora de su sociedad. Con el estudiante que está por egresar, el interno, que ya practica la Psicología, puede servirnos para ver el nivel del joven que, como universidad, sacamos a la sociedad; ¿ es nuestro interno de Psicología, un joven con un claro sentido en su vida, con valores interpersonales como la solidaridad, la inclinación al servicio, el liderazgo, el compromiso con el otro? También queremos encontrar, si a mitad de carrera, existe alguna diferencia en estos aspectos.

Los valores representan un conjunto de apreciaciones firmes y duraderas que determinan y orientan la conducta de los individuos (Lobos, 1992), de ahí que consideremos oportuno revisar los valores interpersonales en una muestra de estudiantes. El tema del sentido de la vida o del propósito en la vida, también es un tema poco revisado en nuestras tesis en comparación con otros aspectos psíquicos (como la inteligencia, la ansiedad, los hábitos de estudio, la autoestima, etc.) y mucho menos estudiado en muestras 
de estudiantes o internos de Psicología. Consideramos que esto abre un campo de interés tanto teórico como práctico. Tener buenas notas o cierto interés por la Psicología no es suficiente para desarrollar un perfil completo, se necesita más. Pensamos que un buen sentido de vida, interés en ser profesional, motivación y amor a la carrera, tener valores como la solidaridad, la empatía, el interés social, entre otros, son aspectos cruciales para formarse como un buen futuro especialista en Psicología.

Ahora, somos conscientes que la familia peruana está en crisis, al igual que la escuela y la sociedad en su conjunto. Los ingresantes a la facultad de Psicología, respiran todo ese hedor que la sociedad produce: anomia, corrupción, violencia, vacío, apatía, además de que todo eso los agarra muy jovencitos (hoy ingresan a las Universidades desde dieciséis años inclusive sino antes). Muchas veces sin saber qué quieren, a dónde van o quiénes son.

Hay pocas investigaciones sobre la salud psicológica y el bienestar en psicólogos. También eso nos llama la atención. No es que debamos ser perfectos y unos ejemplos de la salud para todos, pero ya dentro de la carrera vemos como la realidad nos muestra que, muchas veces, los primeros que necesitan alguna consejería u orientación son los estudiantes. Esta investigación servirá como fuente de información para psicólogos y educadores que trabajan con estudiantes universitarios de carreras como la Psicología. Conocer el nivel de sentido de vida y los valores interpersonales de nuestra muestra de estudiantes universitarios puede servir como termómetro de la realidad psicosocial en la que se mueven muchos estudiantes que dirigen sus intereses en la carrera de Psicología, a veces, pensamos, más para encontrarse y ayudarse ellos mismos que para hacerlo como futuros consultantes, como lo hallado hace años en el trabajo de la psicóloga Nelly Requena, citada por Smith (1985).

Por su parte, desde México, García señala "el gran peligro al que nos enfrentamos los individuos, es a perder la propia identidad y el sentido de la vida; es decir, a caer en la enajenación que nos hace vivir lo que no queremos o no somos: objetos de la propaganda, las modas sociales y los valores huecos" (García, 2001, p. 36). Hoy el sentido de vida en jóvenes es un tema que ha cobrado vigencia y debe ser explorado. Debats (1999; citado por Velasco, 2004) nos comenta que investigadores de diversas disciplinas científicas están poniendo atención al tema del sentido de la vida porque han encontrado que este desempeña un papel relevante en la salud psicológica de las personas así como en la calidad de su existencia. Y como señala Lobos Castillo, "la investigación de los valores y las relaciones interpersonales nos permite conocer que es lo que más valora una persona cuando se relaciona con los demás, y es de importancia ya que todas las actividades humanas se desarrollan dentro de organizaciones que requieren que el sujeto tenga un buen manejo de relaciones interpersonales para obtener una convivencia armónica en las diferentes organizaciones a las que pertenezca" (Lobos, 1992, p. $56)$.

Nos interesa y nos preocupa el estado de los jóvenes estudiantes de la carrera de Psicología, población con la que convivimos y por eso hemos seleccionado una muestra para este estudio y su análisis. Nuestro trabajo nos permitirá detallar qué valores interpersonales presenta la muestra de estudiantes de la carrera Psicología elegida además de conocer su nivel de sentido de vida actual.

\section{OBJETIVOS}

\section{Objetivo General:}

- Determinar la relación existente entre las variables de Sentido de la vida y los Valores Interpersonales en estudiantes de la carrera de Psicología de diferente sexo y año de estudios, de una universidad privada de Lima.

\section{Objetivos específicos:}

- Identificar la existencia de variación en el nivel del sentido de vida que caracteriza a un grupo de estudiantes de la carrera de Psicología.

- Identificar la diferencia en los niveles del sentido de vida entre estudiantes hombres y mujeres de la carrera de Psicología.

- Identificar los valores interpersonales que caracterizan a los estudiantes de la carrera de Psicología. 
- Determinar el grado de relación existente entre el sentido de vida y los valores interpersonales en los estudiantes de la carrera de Psicología.

- Determinar si existen diferencias en la relación del sentido de vida y los valores interpersonales según el sexo.

- Determinar si existen diferencias en la relación del sentido de vida y los valores interpersonales según el nivel de estudios.

\section{POBLACIÓN Y MUESTRA}

La población está conformada por todos los estudiantes matriculados en estudios presenciales de Psicología durante el ciclo 2017-1, en la Facultad de Psicología de una universidad privada de Lima, los cuales suman más de mil personas. De esa población tomaremos, por medio de un muestreo no probabilístico intencional (Kerlinger, 1992), 277 estudiantes de ambos sexos divididos de la siguiente forma:

- NIVEL INICIAL DE LA CARRERA: 103 estudiantes de los ciclos IX y X (realizando su internado - prácticas pre profesionales-) de las tres áreas: clínica, educativa y organizacional

- NIVEL MEDIO DE LA CARRERA: 83 estudiantes de los ciclos V y VI (a mitad de carrera)

-NIVELFINALDELACARRERA:91 estudiantes de los ciclos I y II (primer año de estudios).

La muestra abarcará estudiantes de ambos sexos y de edades que fluctúan entre los 16 a más años. Los requisitos serán que sean estudiantes matriculados en el semestre 2017-1; que Psicología sea su primera carrera; que tengan una asistencia regular a clases y con la disposición de participar el día de la evaluación.

\section{INSTRUMENTOS Y MATERIALES}

Los instrumentos de recolección de información que se emplearán en este trabajo serán:

Cuestionario de valores interpersonales (SIV: Survey of Interpersonal Values) de Leonard V. Gordon, procedencia Science Research Associates - Chicago, Illinois, cuya traducción y adaptación al Perú ha sido realizada por Leonardo Higueras (1972) y Walter Pérez actualizó los baremos en el año 1987.

Midelos valores que se manifiestanen las relaciones con los demás. Estos valores tienen importancia en el ajuste personal, familiar, social y profesional de las personas. Está elaborado con técnicas del análisis factorial y como un cuestionario de elección forzada. Está conformado por un conjunto de proposiciones o juicios ante los cuales se solicita la reacción del sujeto. Consta de 90 ítems o reactivos agrupados en tríadas y en cada tríada el sujeto tiene que elegir el ítem que represente lo que es más importante para él así como la afirmación que representa lo menos importante, de ese modo siempre en cada tríada quedará un ítem en blanco. El cuestionario está distribuido de tal forma que evalúan seis áreas o seis valores. Los cuáles son:

\section{1) Soporte:}

Esta área mide el deseo de ser tratado con comprensión, amabilidad y consideración; recibir apoyo por parte de los demás.

\section{2) Conformidad:}

Esta área mide el deseo de hacer lo que es socialmente correcto y aceptado, siguiendo las normas.

\section{3) Reconocimiento:}

Esta área mide la inquietud de ser admirado y considerado como una persona importante y conseguir el reconocimiento de los demás.

\section{4) Independencia:}

Acá se evalúa el deseo de hacer lo que uno quiere hacer y ser libre para decidir por sí mismo, ser capaz de actuar según criterios propios.

\section{5) Benevolencia:}

Indaga el interés en hacer las cosas por los demás y compartirlas con ellos, ayudar a los poco afortunados y ser generoso.

\section{6) Liderazgo:}

Indaga el interés por hacerse cargo de otras personas y tener autoridad sobre ellas, tener poder.

Los tres ítems de cada tríada fueron igualados hasta donde fue posible en deseabilidad social. Así queda reducida la probabilidad de que el individuo responda de acuerdo a lo que se considera deseable 
socialmente y no según el grado de importancia que realmente tiene cada ítem para él.

El Cuestionario SIV se toma a partir de los 14 años en adelante y puede aplicarse de manera individual como colectiva, si bien no hay tiempo límite bien se puede culminar en un tiempo aproximado de 15 minutos. Puede ser tomado en el consejo psicológico, en orientación vocacional o profesional, en procesos de selección y valoración y, como instrumento de investigación.

\section{- Confiabilidad}

Hay acuerdo general en que la confiabilidad de la prueba es muy buena. Los coeficientes obtenidos con el método test-retest en todas las escalas, varían entre 0,78 y 0,89 . Usando la fórmula de Kuder-Richardon, oscilan entre 0,71 y 0,86 . Dado que los ítems del SIV no se puntúan en forma dicotómica, la confiabilidad por correspondencia interna de cada escala se determina mediante la ecuación llamada "coeficiente alfa", que no es sino una modificación de la fórmula de KuderRichardon. Estos coeficientes de confiabilidad, según opina Gordon, son lo suficientemente altos como para permitir interpretaciones.

\section{- Validez}

El SIV fue desarrollado mediante el uso del análisis factorial. Puede decirse, por lo tanto que las escalas del SIV tienen validez factorial. Otra manera de evaluar la validez de un cuestionario es determinar la razonable relación entre dicho instrumento y otras medidas. $\mathrm{Si}$ estas relaciones corresponden a las expectativas y son lógicas y uniformes, se acumula la confianza adicional respecto a la utilidad práctica de la prueba. En ese sentido el SIV ha sido correlacionado con otras medidas de valores y de rasgos de personalidad como el Inventario de Personalidad de Gordon (IPG) y el Perfil de personalidad de Gordon (PPG) con muy buenos resultados. Podemos decir entonces que el SIV presenta validez y confiabilidad.

Inventario de propósito en la vida (PIL: Purpose in life test) desarrollada por James Crumbaugh y Leonard Matholick; mide qué tanto sentido le encuentra la persona a su vida en el momento de la evaluación. La adaptación en España la realiza Angeles Noblejas de la Flor (1994) y para Lima, Claudine Bartschi (1999).
Está preparada en escalas tipo Likert y consiste en elegir una unidad de medida (valores entre 1 el más bajo y 7 el más alto) a 20 preguntas. La puntuación más alta sería 140 puntos y la más baja, solo 20 puntos. Así:

- Un puntaje de 91 puntos o menos significa una BAJA ORIENTACIÓN AL SENTIDO.

- Un puntaje entre 92 y 111 significa INCERTIDUMBRE.

- Un puntaje de 112 o más significa SENTIDO Y PROPÓSITOS CLAROS EN LA VIDA.

El Cuestionario PIL se toma a partir de los 16 años en adelante y puede aplicarse de manera individual como colectiva, si bien no hay tiempo límite bien se puede culminar en un tiempo aproximado de 10 minutos.

El PIL Test consta de tres partes. En esta investigación solo emplearemos la primera parte. Esta primera está formada por 20 ítems, donde la persona se sitúa a sí mismo en una escala del 1 al 7 entre dos sentimientos extremos, que representa la parte cuantitativa del test. La segunda y tercera parte no se cuantifican (los intentos para lograrlo han añadido poca información a la aportada por la primera parte), lo que justifica su no inclusión en estudios de investigación (según indican Crumbaugh y Matholick, 1969; citados por Cadavid y Díaz, 2008). Su objetivo es proporcionar información para su interpretación clínica. La segunda y tercera parte tienen como objetivo proporcionar información cualitativa para una interpretación de tipo clínica. En la segunda parte se solicita al participante que complete frases sobre el sentido de su vida. Y en la parte tercera, se pide que exprese libremente su situación de vida actual, describiendo en detalle los propósitos, metas y ambiciones así como su progreso respecto a ellos.

\section{- Validez y Confiabilidad}

En Argentina, en el año 2016, Gottfried, realiza una adaptación argentina del PIL Test (Test de Sentido en la Vida) de Crumbaugh y Matholick y luego de su investigación afirma que el PIL Test (Test de Sentido 
en la Vida) versión Argentina 2014, posee buenas propiedades psicométricas por lo que puede ser empleado para evaluar sentido en la vida y su opuesto vacío existencial con un aceptable grado de fiabilidad y validez.

La confiabilidad del PIL fue estudiada por Crumbaugh y Matholick (1964) por medio del método de división por mitades, obteniéndose un coeficiente de correlación de Pearson de 0.81 y coeficiente de 0.90 corregido (método Spearman-Brown). La misma relación fue establecida por Crumbaugh en 1968, obteniendo un coeficiente de correlación de Pearson de 0.85 y de 0.92 corregido (Valdivia, 2007). En Lima, Claudine Bartschi (1999), se titula con su tesis Evaluación del propósito en la vida y la búsqueda de metas noéticas en estudiantes universitarios. Emplea la prueba PIL, halla resultados análogos en la adaptación e indica que cuenta con validez y confiabilidad, por lo que su empleo es acertado.

El PIL ha sido validado en países de varios continentes, teniendo un fuerte acercamiento a los factores que lo componen (Martínez, 2012).

No hay respuestas correctas ni incorrectas en ninguna de las dos pruebas a emplear en esta investigación.

\section{PROCEDIMIENTO}

Luego de los permisos del caso se procedió a la evaluación de la muestra en los momentos en que los estudiantes estaban la Facultad y se encuentren dispuestos. Al ser cuestionarios que no toman mucho tiempo se tomará ambos (SIV y PIL) en un solo momento (tiempo máximo 25 minutos). Se aplicarán las pruebas gradualmente conforme los estudiantes iniciaban sus clases del año universitario 2017. Asíentre enero y febrero se evaluará a los internos (prácticas profesionales, IX y X ciclo) y durante los meses de marzo y abril a los demás estudiantes. Seguidamente se elaborará una base de datos donde se irá analizando los resultados.

Nosotros mismos nos encargamos de la evaluación y la calificación. Nos presentamos y explicamos el motivo de la visita, el objetivo de la investigación y las características de los cuestionarios. La participación fue anónima y se les explicó que no habían respuestas correctas ni incorrectas. Además se indicó muy claramente que la participación era voluntaria y quien no deseaba participar estaba en todo su derecho, Solo se evaluó a los estudiantes animados a participar de la investigación. Se tuvo el cuidado de que los estudiantes hayan entendido perfectamente lo que tenían que hacer, para eso se dieron las instrucciones y estuvimos atentos a cualquier duda que pudiera aparecer entre los estudiantes, aunque las pruebas eran sencillas. Los instrumentos se administraron siguiendo las normas establecidas.

La corrección del SIV se realizará mediante la aplicación de plantillas de corrección directamente sobre las hojas de respuesta. La puntuación por tríada varía entre 2, 1 o 0 puntos. La corrección del PIL se realizará con una suma simple en la misma hoja de respuestas. El valor mínimo será de 20 puntos y el máximo de 140 puntos.

\section{INTERPRETACIÓN Y DISCUSIÓN DE LOS RESULTADOS}

Luego del procesamiento de los datos encontramos que el 41,9\% de los estudiantes de Psicología alcanzan una Alta orientación al sentido de la vida, esto es aproximadamente 4 de cada 10 alumnos, los que tienen metas claras y se encuentran motivados por alcanzar sus posibilidades. Si juntamos los que se encuentran en la Incertidumbre tanto como los que obtienen una Baja orientación al sentido, sumamos más de $58 \%$ de la muestra (aproximadamente 6 de cada 10 alumnos), siendo esto preocupante, pero no sorprendente, por varios factores que hemos estado ya comentando. 
Tabla 1.

Niveles de sentido de la vida

\begin{tabular}{lcccc}
\hline Niveles & Frecuencias & Porcentaje & $\begin{array}{c}\text { Frecuencias } \\
\text { teóricas esperadas }\end{array}$ & Residual \\
\hline Alta orientación al sentido de la vida & & $41.9 \%$ & 92,3 & 23,7 \\
Incertidumbre en cuanto al sentido de la vida & 108 & $39.0 \%$ & 92,3 & 15,7 \\
Baja orientación al sentido de la vida & 53 & $19.1 \%$ & 92,3 & $-39,3$ \\
Prueba Ji cuadrado de bondad de ajuste & Valor & Grados de libertad & Nivel de significación \\
& 25,480 & 2 & p < 0,000 \\
\hline
\end{tabular}

En cuanto al nivel del sentido de la vida y el tiempo de estudios universitarios de Psicología, hay una relación significativa, pues se encuentra que la baja orientación al sentido de la vida tiene a disminuir a medida que los estudiantes llegan al fin de la carrera, y una alta orientación al sentido de la vida tiende a manifestarse más en los últimos años de carrera. Hay pues, mayor sentido de vida en estudiantes de Psicología de ciclos avanzados. Es probable que culminar una carrera universitaria vaya teniendo en ellos un impacto positivo en cuanto a sus objetivos vitales y a su propia sensación de valía. Podemos afirmar que existen diferencias en el nivel del sentido de vida de los estudiantes de Psicología según los niveles de estudio de una universidad privada de Lima.

Tabla 2 .

Sentido de la vida según los niveles de estudio

\begin{tabular}{|c|c|c|c|c|}
\hline \multirow[t]{2}{*}{ Niveles de estudios } & \multicolumn{3}{|c|}{ Niveles de Sentido de la vida } & \multirow[t]{2}{*}{ Total } \\
\hline & $\begin{array}{l}\text { Baja orientación } \\
\text { al sentido de la vida }\end{array}$ & $\begin{array}{l}\text { Incertidumbre } \\
\text { en cuanto al } \\
\text { sentido de la vida }\end{array}$ & $\begin{array}{l}\text { Alta orientación al } \\
\text { sentido de la vida }\end{array}$ & \\
\hline Inicial (I y II Ciclos) & $\begin{array}{r}22 \\
0,7\end{array}$ & $\begin{array}{r}44 \\
1,0\end{array}$ & $\begin{array}{r}37 \\
-1,5\end{array}$ & 103 \\
\hline Medio (Ciclos V y VI) & $\begin{array}{r}25 \\
3,0\end{array}$ & $\begin{array}{r}33 \\
0,2\end{array}$ & $\begin{array}{r}25 \\
-2,6\end{array}$ & 83 \\
\hline Avanzado (Ciclos IX y X) & $\begin{array}{r}6 \\
-3,7\end{array}$ & $\begin{array}{r}31 \\
-1,2\end{array}$ & $\begin{array}{r}54 \\
4,1\end{array}$ & 91 \\
\hline Total & 53 & 108 & 116 & 277 \\
\hline Coeficiente Gamma & $\begin{array}{l}\text { Valor } \\
0,274\end{array}$ & $\begin{array}{r}\text { Nivel de } \\
\mathrm{p}<\end{array}$ & ificación & Coeficiente Gamma \\
\hline
\end{tabular}

Vemos entonces que un poco más del $40 \%$ de los estudiantes tiene una orientación hacia un sentido de vida claro y sólido. Un $20 \%$ (esto es la quinta parte tiene un Bajo nivel de orientación al sentido y otro casi cerca de $40 \%$ de ellos está en una preocupante incertidumbre. Se relaciona esto con lo que hemos estado proponiendo en nuestro marco teórico sobre el sentido de vida y sus vaivenes en la actualidad y guarda relación con lo encontrado en otras investigaciones. Debemos aquí recordar que algunos años atrás, la colega, Claudine Bartschi realizó la investigación "Evaluación del propósito en la vida desde el punto de 
vista de la logoterapia en estudiantes universitarios". La muestra estuvo conformada por 467 estudiantes pertenecientes a facultades de letras y ciencias de una universidad particular de Lima Metropolitana. Los resultados mostraron la presencia de un vacío existencial o falta de sentido en la vida en un 48,6\% de estudiantes. Y en el extranjero, Magaña y cols. (2004) estudiaron el sentido de vida en los estudiantes del primer semestre de la Universidad de La Salle Bajío en la Ciudad de México; en sus resultados se encontró que el $55.5 \%$ de los estudiantes muestran una definición adecuada del Sentido de Vida; el 33.3\% se ubica en la zona de indefinición; y, el 11.2\% muestra falta de Sentido. Está también el trabajo de Martínez y Castellanos (2013) en Colombia, quienes realizaron una investigación de tipo descriptivo transversal y se encontró que 36,7 \% de los participantes (estudiantes de la ciudad de Bogotá) presentaron bajo sentido de vida. Todo esto nos hace pensar en una situación que no es actual y que no está circunscrita a un país o a una sola casa de estudios. También en la ciudad de Madrid, el grupo de Consuelo Rollán y cols. (2005) encontraron que la satisfacción familiar, el bienestar psicológico y el sentido de vida, se relacionan entre sí. Afirman que son tres aspectos relevantes para la vida de los sujetos estudiados, de modo que no es factible tener un alto nivel en uno de los factores estudiados y bajo en los otros, o bien, un nivel muy bajo en uno de los factores y alto en los otros. Esto también lo confirma Maseda y cols. (2011) en su investigación sobre el sentido de vida en los jóvenes españoles, encontrando que las fuentes prioritarias del sentido de vida en la juventud son la familia y los amigos. En su estudio el $72,8 \%$ considera que familia y amigos son referentes con mucho sentido en sus vidas. A tener en cuenta estas otras variables (satisfacción familiar y bienestar psicológico) a la hora de buscar algunas posibles causas de estas cifras halladas en este trabajo.

Sin embargo, se encuentra un mayor nivel de sentido en el grupo de estudiantes de ciclos avanzados (IX y X). Tener unos años más y estar terminando la carrera de Psicología, con todo lo estudiado y experimentado, puede ser la posible causa de este puntaje.

En los valores interpersonales más considerados por los estudiantes de Psicología está el valor de Benevolencia. El autor del inventario SIV considera como benevolencia al interés por hacer las cosas por los demás y compartirlas con ellos, ayudar a los poco afortunados y ser generoso(a). Un nivel alto indica actitud de amor al prójimo, deseo de ayudar a los más necesitados. Esto es un dato importante pues señala que hay una inclinación favorable en los estudiantes de Psicología hacia el prójimo. Recordamos también que en la investigación de Aguilar (2003), citado por Navarro (2009), donde comparó los valores interpersonales en alumnos con Alta y Baja autoestima de los tres primeros ciclos de la Escuela de Psicología de la Universidad Señor de Sipán (Chiclayo), encontró que los estudiantes de estos tres primeros ciclos que poseen alta autoestima poseen también puntajes altos en los valores Liderazgo y Benevolencia.

Tabla 3.

Valores interpersonales característicos de los estudiantes de Psicología (n=277)

\begin{tabular}{lccc}
\hline Valor Interpersonal & Media & Puntaje máximo teórico & Porcentaje \\
\hline Soporte (S) & 13,11 & 30 & 43,70 \\
Conformidad (C) & 15,44 & 30 & 51,47 \\
Reconocimiento (R) & 9,22 & 26 & 35,46 \\
Independencia (I) & 18,88 & 32 & 59,00 \\
Benevolencia (B) & 18,71 & 30 & 62,37 \\
Liderazgo (L) & 14,49 & 30 & 48,30 \\
\hline
\end{tabular}


Como valores interpersonales menos considerados aparecen el Reconocimiento en primer lugar y el Soporte en segundo lugar; esto puede significar poco interés en ser elogiado o reconocido y poca necesidad de apoyo, que creemos puede ir desde cierta autosuficiencia o poca expectativas de elogio y sostén. Podemos afirmar que existen diferencias significativas en los valores interpersonales entre los estudiantes de una universidad privada de Lima.

En cuanto a los valores interpersonales y el nivel de estudios se puede observar que el valor conformismo tiene una media de rangos más elevada en los alumnos del nivel inicial. Este valor representa el deseo de hacer lo que es socialmente correcto y aceptado, siguiendo las normas. De pronto el ser estudiantes más jóvenes y del nivel inicial explica que puntúen más alto aquí.

Encontramos en esta investigación que el sexo, sea hombre o mujer, estadísticamente no fue significativo tanto en su relación directa con el sentido de vida como en su relación directa con los valores interpersonales de los estudiantes de la carrera de Psicología. Es decir cuando analizamos por separado, sexo y sentido de vida, no se encuentra que un grupo tenga más sentido. Este resultado por ejemplo difiere del encontrado en España por García-Alandete y cols. (2011), quienes realizan una investigación donde se analizan las diferencias asociadas al sexo, en las puntuaciones usando el mismo Purpose-In-Life Test (PIL) en un grupo de 309 estudiantes universitarios (207 mujeres y 102 hombres) de edades comprendidas entre 18 y 45 años. En el grupo estudiado y de manera estadísticamente significativa, las mujeres se caracterizan por un nivel general de sentido de la vida superior al de los hombres, por mayor percepción de sentido y valoración de la vida como llena de cosas buenas, emocionantes y razones para ser vivida, por una percepción más positiva del mundo en relación con la propia vida, por tener objetivos y metas ligados a las acciones concretas cotidianas, por un mayor sentido de responsabilidad hacia los objetivos existenciales y por dedicar menos pensamientos al suicidio. Asimismo, son más sensibles a las cosas de la vida cotidiana y les atribuyen más importancia, muestran un mayor sentido de responsabilidad con respecto al día a día y con respecto a las metas y tareas más inmediatas, concretas y específicas.

Tabla 4.

Sentido de la vida y sexo

\begin{tabular}{lcccc}
\hline Sexo & Niveles de Sentido de la vida & & Total \\
& $\begin{array}{c}\text { Baja orientación } \\
\text { al sentido de la vida }\end{array}$ & $\begin{array}{c}\text { Incertidumbre en cuanto } \\
\text { al sentido de la vida }\end{array}$ & $\begin{array}{c}\text { Alta orientación } \\
\text { al sentido de la vida }\end{array}$ \\
\hline Varón & 15 & 18 & 19 & 52 \\
& 1.6 & -0.5 & -0.6 & 225 \\
Mujer & 38 & 90 & 0.3 & 277 \\
Total & -0.8 & 0.2 & 116 & $\begin{array}{c}\text { Nivel de } \\
\text { Significación } \\
\text { Prueba Ji cuadrado }\end{array}$ \\
\cline { 2 - 5 } & 53 & 108 & Palor & Prueba Ji \\
cuadrado
\end{tabular}

En nuestro caso, insistimos, no hay datos estadísticamente significativos que nos hagan pensar en una diferencia importante en el sentido de vida según el sexo. Queremos sí, precisar lo siguiente: en nuestro estudio, la muestra es mayoritariamente femenina y casi quintuplica a la masculina. Actualmente 
es una realidad la gran mayoría de mujeres que estudian Psicología en muchas facultades. Si bien los resultados en cuanto al sexo y el sentido de vida no son estadísticamente significativos, y hemos concluimos que ambas variables (sexo y nivel de sentido de vida) son independientes entre sí, y no existe una mayor relación entre las dos, sin embargo vemos que los estudiantes varones se distribuyen casi por igual entre alto, medio y bajo nivel de orientación al sentido (15, 18 y 19 estudiantes respectivamente, de un total de 52 hombres consultados). No se encuentra eso en las damas, en ellas se refleja la proporción que encontramos para toda la muestra: casi un $20 \%$ con nivel bajo, un $39 \%$ medio y un $41 \%$ aproximadamente con un nivel alto, 38, 90 y 97 estudiantes respectivamente, de un total de 225 mujeres consultadas). Nos arriesgamos a interpretar estos datos parciales como una posible señal de que en las estudiantes mujeres podría haber un mayor nivel de sentido de vida que en comparación con los hombres. De pronto, podría haber mayor compromiso en ellas en estudiar Psicología o ser vista esta tarea como un mayor logro. Una siguiente investigación con una muestra de varones mucho mayor nos ayudaría a confirmar o a rechazar esta conjetura, avisamos, aún apresurada. Asimismo cuando analizamos sexo y valores interpersonales no encontramos ninguna diferencia significativa.

En nuestro trabajo se ve claramente que los valores interpersonales característicos de los estudiantes de Psicología son la Benevolencia, seguido por el de Independencia en tanto que el menos característico es el de Reconocimiento. Siendo esto así, nuestros estudiantes evaluados consideran más importante el amor al prójimo y la libertad para actuar según su criterio, sin esperar mucho reconocimiento quizá por autonomía o llevar una motivación intrínseca consigo. El valor Conformismo es el valor más alto entre los estudiantes del primer año de estudios, luego tiende a bajar considerablemente según se avanza a los estudios medios para luego mantenerse al final de la carrera, algo parecido a lo que encuentra Requena, citada por Smith (1985). El valor Independencia tiende a subir considerablemente con los estudios para luego bajar un poco a medida que se va terminando la carrera, distinto esta vez a lo encontrado por Requena; y en cuanto al valor de Liderazgo tiende a subir con el tiempo de estudios, aquí sí como señala Requena en su propio trabajo.

Sí hemos encontrado correlaciones estadísticas positivas significativas entre la variable sentido de vida y los valores Conformismo y sobre todo Benevolencia, siendo con este último valor donde se obtiene la correlación más alta. Recordemos que Benevolencia involucra interés por el prójimo, y esto sabemos debe estar presente en el perfil del psicólogo, al ser nuestra carrera una de servicio social. Por otra parte, hallamos correlaciones negativas estadísticas muy significativas entre el sentido de vida y los valores interpersonales Soporte y Reconocimiento, siendo este último valor el más elevado negativamente. Esto hace posible aceptar en parte la hipótesis general que dice: "Existe una relación significativa entre el sentido de vida y los valores interpersonales en un grupo de estudiantes de la carrera de Psicología de una universidad privada de Lima".

Tabla 5.

Correlaciones de Sentido de la vida y los valores interpersonales $(n=277)$

\begin{tabular}{lccc}
\hline & Soporte & Conformismo & Reconocimiento \\
Sentido de la vida & $-0,204$ & 0,165 & $-0,221$ \\
Nivel de significación & 0,001 & 0,006 & 0,000 \\
\cline { 2 - 4 } & Independencia & Benevolencia & Liderazgo \\
Sentido de la vida & $-0,110$ & 0,270 & 0,051 \\
Nivel de significación & 0,067 & 0,000 & 0,394 \\
\hline
\end{tabular}


Encontramos una relación positiva entre sentido de vida y Benevolencia, esto es a medida que sube una, tiende a subir la otra; y en parte también lo vemos entre el sentido de vida y el valor Conformismo, aunque en menor grado. También encontramos correlación, aunque negativa, entre sentido de vida y los valores Soporte y Reconocimiento, esto es a mayor sentido de vida los estudiantes muestran menos valor al apoyo externo y a la necesidad de recibir reconocimiento de los demás.

Tabla 6.

Correlación de Sentido de la vida y Valores interpersonales según niveles de estudios

\begin{tabular}{llccr}
\hline Nivel de estudios & & Valores interpersonales & \\
& & Soporte & Conformismo & Reconocimiento \\
Inicial (I-II ciclos) & Sentido de la vida & $-0,171(\mathrm{p}<0,084)$ & $0,288(\mathrm{p}<0,003)$ & $-0,245(\mathrm{p}<0,013)$ \\
Medio (V-VI ciclos) & Sentido de la vida & $-0,165(\mathrm{p}<0,136)$ & $-0,040(\mathrm{p}<0,718)$ & $-0,264(\mathrm{p}<0,016)$ \\
Avanzado (IX y X) & Sentido de la vida & $-0,245(\mathrm{p}<0,019)$ & $0,235(\mathrm{p}<0,025)$ & $-0,087(\mathrm{p}<0,410)$ \\
\cline { 2 - 5 } Nivel de estudios & & Valores interpersonales & Liderazgo \\
& & Independencia & Benevolencia & $0,141(\mathrm{p}<0,154)$ \\
Inicial (I-II ciclos) & Sentido de la vida & $-0,244(\mathrm{p}<0,013)$ & $0,210(\mathrm{p}<0,033)$ & $0,057(\mathrm{p}<0,610)$ \\
Medio (V-VI ciclos) & Sentido de la vida & $0,002(\mathrm{p}<0,984)$ & $0,374(\mathrm{p}<0,000)$ & $-0,070(\mathrm{p}<0,512)$ \\
Avanzado (IX y X) & Sentido de la vida & $-0,047(\mathrm{p}<0,656)$ & $0,156(\mathrm{p}<0,141)$ &
\end{tabular}

También existe una correlación negativa entre el sentido de vida y el valor de soporte, sobre todo en el nivel avanzado de estudios (a mayor sentido de vida, menos necesidad de soporte) y correlación positiva entre el sentido de vida y el conformismo (a mayor sentido de vida, mayor conformismo, y viceversa) y esto más alto aunque ligeramente en estudiantes varones. También se halla una correlación negativa muy significativa, en ambos sexos, entre el sentido de vida y el reconocimiento (a medida que aumenta el sentido de vida, disminuye la necesidad de reconocimiento, y viceversa). Se demuestra que en ninguno de los sexos existe correlación entre el sentido de la vida y el liderazgo. Se halla también una correlación positiva muy significativa, en ambos sexos, entre el sentido de vida y el valor de benevolencia (a medida que aumenta uno, también aumenta el otro, y viceversa). Por esto mencionado, podemos aceptar en parte la hipótesis específica 1 que dice: "El sexo de los estudiantes influye en la correlación del sentido de vida y de los valores interpersonales, en los estudiantes de Psicología de una universidad privada de Lima”.

Tabla 7.

Correlación de Sentido de la vida y Valores interpersonales según el sexo

\begin{tabular}{llccc}
\hline Sexo & & Valores interpersonales & \\
& & Soporte & Conformismo & Reconocimiento \\
Varón & Sentido de la vida & $-0,237(\mathrm{p}<0,091)$ & $0,150(\mathrm{p}<0,289)$ & $-0,308(\mathrm{p}<0,026)$ \\
Mujer & Sentido de la vida & $-0,197(\mathrm{p}<0,003)$ & $0,164(\mathrm{p}<0,014)$ & $-0,203(\mathrm{p}<0,002)$ \\
\cline { 2 - 5 } Sexo & & & Valores interpersonales & \\
& & Independencia & Benevolencia & Liderazgo \\
Varón & Sentido de la vida & $-0,294(\mathrm{p}<0,034)$ & $0,494(\mathrm{p}<0,000)$ & $0,084(\mathrm{p}<0,552)$ \\
Mujer & Sentido de la vida & $-0,054(\mathrm{p}<0,417)$ & $0,208(\mathrm{p}<0,002)$ & $0,039(\mathrm{p}<0,562)$ \\
\hline
\end{tabular}


Se observa que no existe correlación entre el sentido de vida y los valores de liderazgo e independencia en casi todos los niveles de estudios. Pero con relación al valor interpersonal de Soporte, encontramos una correlación negativa muy significativa, en el nivel avanzado de estudios, esto es que a mayor sentido de vida menos soporte. Similar situación ocurre con el Reconocimiento, principalmente debido a las correlaciones negativas significativas en los niveles inicial (I y II ciclos) y medio (V y VI ciclos).

Además existe una correlación positiva significativa entre el sentido de vida y la Benevolencia en gran parte de los estudiantes. Así pues, podemos aceptar en parte la hipótesis específica 2 que dice: "El nivel de estudios influye en la correlación del sentido de vida y los valores interpersonales en los estudiantes de la carrera de Psicología de una universidad privada de Lima".

\section{CONCLUSIONES}

1. Con esta investigación, hemos encontrado que existe una problemática alarmante. Muchos jóvenes realizando estudios universitarios sin un definido sentido de sus vidas. Encima de esto, la población evaluada está estudiando Psicología, una carrera que exige contar con un perfil básico necesario para un efectivo trabajo de agentes de cambio de una sociedad como la nuestra que no se caracteriza por andar bien; los niveles de violencia y de corrupción demuestran que en temas de salud psicológica andamos aún atrasados.

2. Desde hace décadas grandes autores vienen estudiando el tema del sentido y el vacío existencial así como la dimensión axiológica y ética en las personas. El ser humano no puede prescindir de estos aspectos, y en ese sentido, este trabajo ha intentado indagar como están un grupo de jóvenes estudiantes de Psicología de una Universidad Privada de Lima, en aspectos como el sentido de sus vidas y los valores interpersonales.

3. Se encuentra que solo un $41,9 \%$ de la muestra obtiene un puntaje de alta orientación al sentido en sus vidas. Podemos decir que estos niveles de sentido de vida tienen a aumentar con el paso de los años de estudio y el avance en la formación de la carrera. No existe en nuestra investigación, una relación estadísticamente significativa entre el nivel del sentido de vida y el sexo en los estudiantes de la carrera de Psicología.

4. Por otro lado, hay un porcentaje marcadamente superior en el valor interpersonal Benevolencia entre los estudiantes de la carrera de Psicología $(62,37 \%)$, así vemos que hay una inclinación favorable en los estudiantes evaluados a servir al prójimo y ayudarlos, aspectos siempre presentes en un psicólogo.

5. Existe una tendencia pequeña pero significativa a que a medida que suben las puntuaciones en el sentido de la vida, suben las puntuaciones en el valor Benevolencia $(r=0,270)$ y Conformismo $(r=0,165)$. Y a medida que bajan las puntuaciones en sentido de vida, bajan también las puntuaciones en estos dos valores interpersonales. Se observa también, una tendencia estadísticamente significativa por la cual a medida que suben las puntuaciones en sentido de vida, bajen las puntuaciones en Soporte $(r=-0,204)$ y Reconocimiento $(\mathrm{r}=-0,221), \mathrm{y}$ viceversa.

6. En la investigación se encuentra que el nivel de estudios y el sexo influyen en parte, en la correlación entre el sentido de vida y los valores interpersonales.

7. Luego de procesar debidamente los resultados obtenidos, podemos concluir que se acepta en parte la hipótesis general que dice: "Existe una relación significativa entre el sentido de vida y los valores interpersonales en un grupo de estudiantes de la carrera de Psicología de una universidad privada de Lima". El valor interpersonal Benevolencia es el de más alta correlación positiva $(r=0,270)$.

8. Podemos aceptar en parte la hipótesis específica que dice: "El sexo de los estudiantes influye en la correlación de sentido de vida y los valores interpersonales". En algunos valores interpersonales, el sexo influye más que en otros, como en el caso de la Benevolencia (varones $r=0,494$, mujeres $r=0,208$ ); Independencia (varones $r=-0,294$, mujeres $r=-0,054$ ); Reconocimiento (varones $r=-0,308$, mujeres $r=-0,203$ ) y Soporte (varones $r=-0,237$, mujeres $r=-0,197$ ).

9. También podemos aceptar en parte la hipótesis específica que dice: "El nivel de estudios de los alumnos influye en la correlación del sentido de vida 
y los valores interpersonales". Igualmente, en algunos valores interpersonales, el nivel de estudios influye más que en otros, como en el caso de Benevolencia con niveles de correlación más significativos en el nivel inicial $(r=0,210)$ y medio $(r=0,374)$; en el valor Reconocimiento con valores en el nivel inicial de $\mathrm{r}=$ $-0,245$ y medio de $r=-0,264$. En el valor interpersonal de Conformismo la correlación es significativa en el nivel inicial con un $r=0,288$ y en el nivel avanzado con un $r=0,235$; mientras que en el valor Soporte encontramos una correlación negativa en el nivel avanzado $(r=-0,245)$.

\section{SUGERENCIAS INVESTIGACIÓN}

LUEGO

DE

LA

1. De pronto, es necesario realizar una ampliación de esta misma investigación en muestras mucho más extensas para confirmar o rechazar las tendencias encontradas en esta investigación. Además sería interesante realizar trabajos comparativos entre distintas casas de estudios, tanto privadas como públicas, de Lima como del interior del país. Esperamos poder hacerlo en los próximos años.

2. Pensamos que las facultades de Psicología, en especial, debieran permitir contar dentro de los estudios en la carrera de Psicología, con un tiempo y un espacio para actividades de crecimiento y desarrollo personal, más a la luz de que la mayoría de los jóvenes estudiantes, muchos de ellos aún adolescentes, no tienen una clara orientación al sentido de la vida ni propósitos claros.

3. Estar atentos, como psicólogos y docentes, a los estímulos nocivos que nuestra sociedad produce y denunciarlos así como analizarlos y discutirlos en foros, clases y espacios académicos. Necesitamos una sociedad que promueva valores y no antivalores, esperanza y no desesperanza, orden y no caos, todo esto solo contribuye negativamente a un mayor vacío y desorientación entre los más jóvenes.

4. Mejorar los procesos de selección universitaria. Aparentemente, están postulando estudiantes que no saben muy bien porque llegan a la Universidad y no tienen muy claro su papel y su sentido de vida. Estudiantes así, es probable, no solo no destaquen académicamente sino que pierdan valioso tiempo que bien podrían invertir en un proceso previo de orientación y/o terapia, inclusive.

5. Se sugiere realizar mayores investigaciones en cuanto al tema del sentido de la vida. Son pocas las investigaciones en nuestro medio que manejan esta importante variable de los propósitos en la vida de las personas. Y más aún en estudiantes de carreras como la Psicología.

6. Desde acá aprovechamos también, para exigir a las autoridades nacionales que haya una mayor inversión en temas de salud psicológica. Es urgente en el país el trabajo no solo en atención primaria sino en prevención y de promover una cultura de respeto, de paz y de posibilidades.

\section{REFERENCIAS}

Álvarez de Spano, M.E. (2015). Vivir la Logoterapia. (2da. Edición). Argentina: Editorial Dunken.

Arboccó, M. y O’Brien, J. (2012). Impacto de la televisión basura en la mente y la conducta de niños y adolescentes. Avances en Psicología, 2, 43-57. [Lima: Universidad Femenina del Sagrado Corazón]

Arboccó, M. (2014). La importancia del proyecto de vida. Diario Oficial El Peruano, p. 12, 27 de diciembre del 2014.

Arboccó, M. (2015a). Psicología, psicoterapia y sociedad. Reflexiones Humanístico Existenciales. Avances en Psicología, 1. 49-60. [Lima: Universidad Femenina del Sagrado Corazón]

Arboccó, M. (2015b). ¿Quién es el ser humano? Diario Oficial El Peruano, p. 12, 29 de mayo del 2015.

Arboccó, M. (2016a). “ ¿Nosotros, estamos sanos? La Salud Psicológica en los psicólogos”. Conferencia presentada en el XIII Congreso Nacional de Profesionales y Estudiantes de Psicología, organizado por la Facultad de Psicología de la Universidad Inca Garcilaso de la Vega. Lima - Perú: 23 de Noviembre del 2016.

Arboccó, M. (2016b). De las neurosis histéricas del siglo XIX al vacío existencial actual. Lima: 
Diario Oficial El Peruano, p. 12, 15 de diciembre del 2016.

Arboccó, M. (2016c). La importancia de la salud psicológica. Lima: Diario Oficial El Peruano, p. 12, 23 de noviembre del 2016.

Arboccó, M. (2017). La sociedad de lo descartable. Lima: Diario Oficial El Peruano, p. 12, 12 de mayo del 2017.

Baggini, J. (2004). El sentido de la vida. Y las respuestas de la Filosofía. España: Urano.

Bartschi, C. (1999). Evaluación del propósito en la vida y la búsqueda de metas noéticas desde el punto de vista de la Logoterapia em estudiantes universitários. Tesis para obtener la licenciatura em Psicología. Lima: Universidad de Lima.

Bauman, Z. (2007). Vida de consumo. Argentina: Fondo de Cultura Económica.

Cadavid, M.V. y Díaz, V.M. (2008). Tipos de sentido de vida en jóvenes universitarios. Tesis para obtener el grado de Maestro en Educación. Colombia: Universidad de La Sabana.

Camacho, S. (2002). Relación entre la percepción del tipo de família y los valores interpersonales em adolescentes de cuarto y quinto grados de Secundaria de Lima-Cercado. Tesis para optar el Grado Académico de Magister en Psicología. Lima: Universidad Nacional Mayor de San Marcos.

Campos, H. (2016). Sentido de vida en un grupo de jóvenes de un centro de rehabilitación en adicciones de Lima Metropolitana. Tesis para optar el título de Licenciada en Psicología, Lima: Universidad de Ciencias Aplicadas (UPC). Recuperado de:http://repositorioacademico.upc. edu.pe/upc/bitstream/10757/620675/1/campos_ hm.pdf

Clark-Carter, D. (2002) Investigación cuantitativa en Psicología. México: Oxford.

Coolican, H. (2005). Métodos de investigación y estadística em Psicología. Tercera edición. México: Manual Moderno.

DiCaprio, N. (1984). Teoría de la Personalidad. México: Nueva Editorial Interamericana.
Elorza, H. (1987). Estadística para ciencias del comportamiento. México, D.F.: Harla.

Escudero, J. (2007). Relación entre resiliencia y sentido de vida (religiosidade intrínseca y objetivos vitales) em adolescentes de cuarto y quinto de Secundaria. Tesis para optar el título profesional de psicólogo. Lima: Universidad Nacional Mayor de San Marcos.

Fabry, J. (2009). Señales del camino hacia el sentido. Descubriendo lo que realmente importa. México: LAG.

Frankl, V. (2004). El hombre en busca de sentido. España: Herder.

Frankl, V. (2008). Ante el vacío existencial. Hacia uma humanización de la psicoterapia. España: Herder.

Fromm, E. (1964). Psicoanálisis de la Sociedad Contemporánea. Hacia una sociedad sana. Sexta. Edición. México: Fondo de Cultura Económica.

García-Alandete, J., Martínez, E. R., Soucase Lozano, B. \& Gallego-Pérez, J. F. (2011). Diferencias asociadas al sexo en las puntuaciones total y factoriales del Purpose-In-Life Test en universitarios españoles. En Universitas Psychologica, 10 (3), 681-692.

García-Alandete, J., Gallego-Pérez, J.F. y PérezDelgado, E. (2009). Sentido de la vida y desesperanza: un estudio empírico. En Univ. Psychol. Bogotá, Colombia. Vol. 8, No. 2, págs. 447-454.

García, C. (2010). La vida es una moneda. El arte de vivir. Barcelona: Plataforma Editorial.

García, E. (2001). Calidad de vida en el estudiante universitario. Tesis para obtener el grado de Maestro en Ciencias de la Adminitración. México: Universidad Autónoma de Nuevo León.

Gottfried, A. E. (2016). Adaptación argentina del PIL Test (Test de Sentido en la Vida) de Crumbaugh y Maholick [en línea], Revista de Psicología, 12(23).

Grimaldo, M.P. (1999). ¿Hacia dónde van nuestros jóvenes? Lima: Universidad de San Martín de Porres. Escuela Profesional de Psicología. 
Hernández, R., Fernández, C. y Baptista, P. (2006). Metodología de la investigación. Cuarta Edición. México: McGraw-Hill Interamericana.

Kerlinger,F.(1992). Investigación del comportamiento. Segunda edición en español. México: McGrawHill Interamericana.

Larsen, R y Buss, D. (2005). Psicología de la personalidad.Dominios de conocimiento sobre la naturaleza humana. México: Editorial Mc Graw Hill.

Lipovestky, G. (2000). La era del vacío. Ensayos sobre el individualismo contemporáneo. Décimotercera edición. Barcelona: Anagrama.

Loaiza, O. (2005). Construcción del sentido de vida en jóvenes universitarias. Tesis para obtener el grado de Doctora en Desarrollo Humano. México: Universidad Iberoamericana.

Lobos Castillo, M. (1992). Relación entre los valores interpersonales y el rendimiento escolar em um grupo de adolescentes de cuarto y quinto de secundaria. Tesis para optar el título profesional de psicóloga. Lima: Universidad Nacional Mayor de San Marcos.

Maseda, P. y cols. (2011). El sentido de vida en los jóvenes: redes sociales, relaciones significativas y actividades de ocio. En Revista de Estudios de Juventud. $\mathrm{N}^{\circ} .95$, diciembre, 59- 72. España: Grupo de Investigación PSICOSOC. Centro de Estudios Superiores Don Bosco, adscrito a la Universidad Complutense de Madrid.

Magaña, L.; Zavala, M.A; Ibarra, I.; Gómez Medina, M.T. y Gómez Medina, M.M. (2004). El Sentido de vida en Estudiantes de Primer Semestre de la Universidad de la Salle Bajío. En Revista del Centro de Investigación. Universidad La Salle, Vol. 6, Núm. 22, julio-diciembre, pp. 5-13. [Distrito Federal, México: Universidad La Salle.]

Manrique, H. (2011). Descripción del sentido de la vida en adolescentes infractores de la ciudad de Medellín, En Revista Colombiana de Ciencias Sociales. 2 ( 2) 113-138.

Martinez, E. (2012). El diálogo socrático en la psicoterapia. Colombia: SAPS (Sociedad para el Avance de la Psicoterapia Centrada em el Sentido).
Martínez, E., Trujillo Cano, A. y Trujillo, C.A. (2012). Validación del Test de Propósito Vital (PIL test - purpose in life test) para Colombia. En Revista Argentina de Clínica Psicológica, Vol. XXI, núm. 1,pp. 85-93. [Buenos Aires, Argentina: Fundación Aiglé.]

Martínez, E. (2013). Buscando el sentido de la vida. Colombia: Fundación Coletivo Aquí y ahora.

Martínez, E y Castellanos, C. (2013). Percepción de sentido de vida en universitarios colombianos. En Pensamiento Psicológico. 11 (1) enero-junio, 71-82. Cali, Colombia: Pontificia Universidad Javeriana.

Matos, O. (Set. 2014). Náufragos de la modernidad. En entrevista con Ana Paula Orlandi, Traducción: Katerina Valdivia Bruch. Copyright: GoetheInstitut Brasilien. Recuperado de: http: // www.goethe.de/ins/co/es/bog/kul/mag/fok/ zei/13268288.html, el 20 de diciembre del 2014.

May, R. (2000). El dilema del hombre. Respuestas a los problemas del amor y de la angustia. España: Gedisa Editorial.

Navarro, V. (2009). Valores interpersonales y juicio moral em estudiantes de dos universidades particulares de Lima - Metropolitana. Tesis para optar el Grado Académico de Magister en Psicología. Lima: Universidad Nacional Mayor de San Marcos.

Piaget, J. (1992). Seis estudios de Psicología. Perú: Edición Vlacabo.

Polasek, D. (2002). SPV, Cuestionario de Valores Personales de L.V. Gordon. Su normalización en um grupo de adolescentes de colegios de clase media de Guatemala. Tesis para optar el título de Licenciada em Psicología Clínica. Guatemala: Universidad Francisco Marroquín.

Ponce, H. (2001). Imágenes críticas de la televisión actual. La función social de los medios de comunicación. Lima: Universidad de San Martín de Porres. Escuela Profesional de Ciencias de la Comunicación.

Real Academia Española. (2017). Diccionario de la Lengua Española. Vigésima Tercera Edición. Edición del Tricentenario. Versión virtual. 
Rollán, C., García-Bermejo, M. y Villarrubia, I. (2005). Relación entre la satisfacción familiar, el bienestar psicológico y el sentido de la vida. Págs. 141-150, España: CES Don Bosco.

Salomon, A. (2015). Encontrando y realizando sentido. Diálogo socrático y ejercicios vivenciales en logoterapia. Lima: Universidad Femenina del Sagrado Corazón. Ensayos em Psicología.

Sánchez Paredes, R. (2013). Valores interpersonales y autoconcepto em estudiantes universitários de la carrera de ingeniería ambiental y administración de empresas de uma universidad estatal. Tesis para optar el Grado Académico de Magister en Psicología. Lima: Universidad Nacional Mayor de San Marcos.

Sartori, G. (2004). Homo videns: la sociedad teledirigida. España: Taurus.

Savater, F. (2007). Las preguntas de la vida. Barcelona: Ariel.

Smith Rosazza, C. (1985). Los valores interpersonales del estudiante de Psicología a través de su formación profesional. Tesis para optar el Grado Académico de Bachiller en Psicología. Lima: Universidad Nacional Mayor de San Marcos.

Tueros Cárdenas,R.(2012).Losvaloresinterpersonales y su relación com los estilos atributivos em estudiantes de quinto grado de secundaria. Tesis para optar el Grado Académico de Doctor en Psicología. Lima: Universidad Nacional Mayor de San Marcos.

Valdivia, A. (2007). Vacío existencial y riesgo suicida em pacientes com transtornos de personalidade borderline. En Revista Persona. Vol. 10; páginas 179-197

Velasco, S. (2004). El sentido de vida de los adolescentes: un modelo centrado en la persona. Tesis para obtener el grado de Maestra en Desarrollo Humano. México, Universidad iberoamericana.

Fecha de recepción: 6 de febrero, 2018

Fecha de aceptación: 8 de marzo, 2018 